\title{
NORMAL FAMILIES OF SUBHARMONIC FUNCTIONS ${ }^{1}$
}

\author{
MAYNARD G. ARSOVE
}

1. Introduction. The theory of normal families, long recognized as an invaluable tool in dealing with analytic and meromorphic functions, has been applied by Montel [11] and others to the study of harmonic functions. In each case the methods owe a large portion of their success to the existence of simple conditions ensuring normality, for example, the condition that the functions be locally uniformly bounded. However, the usual conditions on families of harmonic and analytic functions are inadequate to yield normality for families of subharmonic functions. Roughly speaking, subharmonic functions lack the cohesiveness of harmonic and analytic functions.

Since harmonic functions can be regarded as subharmonic functions with identically vanishing mass distributions, one is led to conjecture that some less stringent restriction on the mass distributions will give rise to sufficient cohesiveness. Our principal result is the verification of this conjecture. We show, in fact, that a certain locally uniform condition on the growth of the mass distributions, together with local uniform boundedness of the functions, will result in normality of a family of subharmonic (and, more generally, $\delta$-subharmonic ${ }^{2}$ ) functions.

A special case is the following: if the $\delta$-subharmonic functions are locally uniformly bounded and have mass distributions given by locally uniformly bounded density functions, then the family is normal. Applications are made to certain $L^{p}$ spaces of continuous $\delta$-subharmonic functions, in particular to the construction of a reproducing kernel [1] for the case of $p=2$. Further applications, to functions of the extended class [7] in the theory of several complex variables, will appear in a later paper.

2. The mass restriction. Sufficient conditions for normality and compactness. It should first be explained that we use normality in the finite sense. A family $\mathcal{F}$ of functions on a region $\Omega$ is normal if

Presented to the Society, May 18, 1955; received by the editors April 18, 1955.

${ }^{1}$ The research reported upon here was supported (in part) by the National Science Foundation.

2 For brevity, functions representable as differences of subharmonic functions will be referred to as $\delta$-subharmonic; an introduction to the theory of such functions can be found in [3]. 
every sequence of functions in $\mathcal{F}$ contains a subsequence converging uniformly on compact subsets of $\Omega$. The family is then compact if the limit functions all belong to $\mathcal{F}$. For a development of the fundamentals of the theory of normal and compact families, the results of which we use without explicit reference, the reader is referred to [16].

In the sequel we deal only with $\delta$-subharmonic functions on a plane region $\Omega$, but the transition to $n$-dimensional Euclidean space and to more general spaces (such as Riemann surfaces) presents no special difficulties.

For $w$ any function $\delta$-subharmonic on $\Omega$ we shall denote by $\Phi_{w}^{z}(r)$ the total variation of the mass distribution for $w$ on the neighborhood $N_{r}(z)$ of radius $r$ about $z$. Also, we use $\Omega_{r}$ to signify the set of all points $z$ for which the closure of $N_{r}(z)$ lies in $\Omega$, it being always assumed that $r$ is taken small enough to ensure that $\Omega_{r}$ is non-empty. With these conventions, $\Phi_{w}(r)$ and $\Psi_{w}(r)$ are defined in the extended sense as

$$
\Phi_{w}(\boldsymbol{r})=\sup _{\boldsymbol{s} \in \Omega_{\boldsymbol{r}}} \Phi_{w}^{2}(\boldsymbol{r})
$$

and

$$
\Psi_{w}(r)=\sup _{s \in \Omega_{r}} \int_{0}^{r} \Phi_{w}^{s}(t) d \log t .
$$

Then, if $\mathcal{F}$ is any family of functions $\delta$-subharmonic on $\Omega, \Phi \mathcal{F}(r)$ and $\Psi \mathcal{F}(r)$ are defined as the least upper bounds of $\Phi_{w}(r)$ and $\Psi_{w}(r)$, respectively, as $w$ varies over $\mathcal{F}$.

It turns out that families $\mathcal{F}$ of $\delta$-subharmonic functions satisfying the uniform mass restriction $\lim _{r \rightarrow 0} \Psi \mathcal{F}(r)=0$ behave in many ways like families of harmonic functions.

THEOREM 1. Let $\mathcal{F}$ be a family of functions $\delta$-subharmonic on $\Omega$ such that $\mathcal{F}$ is bounded and $\lim _{r \rightarrow 0} \Psi \mathcal{F}(r)=0$. Then

(1) $\mathcal{F}$ is a normal family,

(2) all functions in $\mathcal{F}$ can be expressed as differences of continuous subharmonic functions, and

(3) the mass distributions for functions in $\mathcal{F}$ have total variations uniformly bounded on compact subsets of $\Omega$.

To prove this, we start with the expression

$$
w(z)=\mu_{r} w(z)+\int_{N_{r}(z)} \log \frac{r}{|z-\zeta|} d m(\zeta),
$$

where $w$ is any function $\delta$-subharmonic on $\Omega, m$ is the mass distribu- 
tion for $w, N_{r}(z)$ is any neighborhood having closure in $\Omega$, and $\mu_{r} w(z)$ is the mean of $w$ over the circumference of $N_{r}(z)$. Note that (2.1) is just the evaluation at $z$ of the Riesz representation for $w$ on $N_{r}(z)$ as a Green's potential plus a harmonic function.

Denoting by $\alpha_{r} w(z)$ the mean of $w$ over $N_{r}(z)$, we find from (2.1) that

(2.2) $w(z)=\alpha_{r} w(z)+\frac{2}{r^{2}} \int_{0}^{r} \rho \int_{N_{\rho}(z)} \log \frac{\rho}{|z-\zeta|} d m(\zeta) d \rho$.

It is evident, moreover, that the second term of the right-hand member is in absolute value no larger than

$$
\int_{N_{r}(z)} \log \frac{r}{|z-\zeta|}|d m(\zeta)|=\int_{0}^{r} \log \frac{r}{t} d \Phi_{w}^{z}(t) .
$$

We now suppose $w$ to belong to $\mathcal{F}$ and use the finiteness of $\Psi_{w}(r)$ to infer that $w$ has no concentrated mass at $z$. There follows

$$
\lim _{t \rightarrow 0} \Phi_{w}^{2}(t) \log t=0
$$

so that an integration by parts yields

$$
\int_{0}^{r} \log \frac{r}{t} d \Phi_{w}^{z}(t)=\int_{0}^{r} \Phi_{w}^{z}(t) d \log t \leqq \Psi \mathcal{F}(r)
$$

Hence,

$$
\left|\frac{2}{r^{2}} \int_{0}^{r} \rho \int_{N_{\rho}(z)} \log \frac{\rho}{|z-\zeta|} d m(\zeta) d \rho\right| \leqq \Psi \mathcal{F}(r) .
$$

It is an immediate consequence of (2.2) and (2.3) that every $w$ in $\mathcal{F}$ is continuous on $\Omega$; in fact, $\alpha_{r} w$ is continuous and we have

$$
\left|w\left(z_{1}\right)-w\left(z_{2}\right)\right| \leqq\left|\alpha_{r} w\left(z_{1}\right)-\alpha_{r} w\left(z_{2}\right)\right|+2 \Psi \mathcal{F}(r)
$$

for $z_{1}$ and $z_{2}$ on $\Omega_{r}$. The continuity of $w$ in turn forces $\alpha_{r} w$ to be continuously differentiable and furnishes the identities [14, p. 11]

$$
\begin{aligned}
& \frac{\partial \alpha_{r} w}{\partial x}=\frac{1}{\pi r} \int_{0}^{2 \pi} w\left(z+r e^{i \theta}\right) \cos \theta d \theta, \\
& \frac{\partial \alpha_{r} w}{\partial y}=\frac{1}{\pi r} \int_{0}^{2 \pi} w\left(z+r e^{i \theta}\right) \sin \theta d \theta .
\end{aligned}
$$

Thus, if $M$ is an upper bound for all $|w|$, we see from the mean-value theorem that 


$$
\left|\alpha_{r} w\left(z_{1}\right)-\alpha_{r} w\left(z_{2}\right)\right| \leqq \frac{4 M}{r}\left|z_{1}-z_{2}\right|
$$

holds for all $w$ in $\mathcal{F}$ and all $z_{1}, z_{2}$ on any neighborhood $\omega$ contained in $\Omega_{r}$.

Combining (2.4) and (2.5) results in

$$
\left|w\left(z_{1}\right)-w\left(z_{2}\right)\right| \leqq \frac{4 M}{r}\left|z_{1}-z_{2}\right|+2 \Psi \mathcal{F}(r),
$$

which implies equicontinuity of $\mathcal{F}$ on $\omega$. By Ascoli's theorem it follows that $\mathcal{F}$ is normal on $\Omega$.

Assertion (2) is established by applying (2.4) to the subharmonic functions occurring in any canonical representation $[3$, p. 331] of $w$. Finally, to show that the total variations of the mass distributions are uniformly bounded on compact subsets of $\Omega$, we observe that

$$
2 \Psi \mathcal{F}(2 r) \geqq \int_{0}^{2 r} 2 \log \frac{2 r}{t} d \Phi_{w}^{2}(t) \geqq \int_{0}^{r} 2 \log \frac{2 r}{t} d \Phi_{w}^{2}(t) \geqq \Phi_{w}^{2}(r)
$$

and cover any given compact subset by a finite number of neighborhoods $N_{r}(z)$. This completes the proof.

We turn next to the examination of the limit of a convergent sequence of functions in $\mathcal{F}$. In the subharmonic case uniformity of the convergence on compact subsets makes the limit function subharmonic, and this of course holds for $\mathcal{F}$ any normal family of such functions. Assuming $\mathcal{F}$ to satisfy the requirements of Theorem 1 , we obtain a corresponding result in the general case.

THEOREM 2. Let $\mathcal{F}$ be as in Theorem 1 , and let $\left\{w_{n}\right\}$ be a sequence of functions in $\mathcal{F}$ converging to a function w on $\Omega$. Then

(1) $w$ can be expressed as the difference of two continuous subharmonic functions,

(2) the mass distributions for $\left\{w_{n}\right\}$ converge vaguely to that for $w$, and

(3) $\Phi_{w}(r) \leqq \Phi \mathcal{F}(r)$ and $\Psi_{w}(r) \leqq \Psi \mathcal{F}(r)$.

PROoF. Since $\left\{w_{n}\right\}$ converges to $w$ uniformly on compact subsets of $\Omega$ and the total variations of the mass distributions are uniformly bounded on these subsets, Theorem 23 of [3] implies that $w$ is almost $\delta$-subharmonic and that the mass distributions for $\left\{w_{n}\right\}$ converge

${ }^{8}$ That is, if $m_{n}$ and $m$ are the corresponding mass distributions, then $\lim _{n \rightarrow \infty} \int f d m_{n}$ $=\int f d m$ for all continuous functions $f$ supported by compact subsets of $\Omega$. This is equivalent to weak convergence of $\left\{m_{n}\right\}$ to $m$ on all bounded subregions with closures in $\Omega$ (see $[3$, p. 345]). 
vaguely to that for $w$. As observed in Lemma 1 of [4], the total variation functionals then exhibit the lower semi-continuity property

$$
\Phi_{w}^{z}(r) \leqq \liminf _{n \rightarrow \infty} \Phi_{w_{n}}^{z}(\boldsymbol{r}) \leqq \Phi \mathcal{F}(\boldsymbol{r})
$$

Hence, by Fatou's lemma

$$
\Psi_{w}^{z}(\boldsymbol{r})=\int_{0}^{r} \Phi_{w}^{z}(t) d \log t \leqq \liminf _{n \rightarrow \infty} \int_{0}^{r} \Phi_{w_{n}}^{z}(t) d \log t \leqq \Psi \mathcal{F}(\boldsymbol{r}) .
$$

This allows us to prove (1) by much the same method as used in proving (2) of Theorem 1 , and our theorem is established.

Theorem 2 leads at once to sufficient conditions for a family of $\delta$-subharmonic functions to be compact.

CoROllary 2.1. Let there be given a constant $M \geqq 0$ and a function $\eta \geqq 0$ such that $\lim _{r \rightarrow 0} \eta(r)=0$. Then the family $\mathcal{F}$ of all $\delta$-subharmonic functions w on $\Omega$ satisfying

$$
\text { (1) } \quad|w| \leqq M \quad \text { and } \quad(2) \quad \Psi_{w} \leqq \eta
$$

is compact.

CoROLlaRy 2.2. Let there be given a constant $M \geqq 0$ and a function $\xi \geqq 0$ such that

$$
\lim _{r \rightarrow 0} \int_{0}^{r} \xi(t) d \log t=0 .
$$

Then the family $\mathcal{F}$ of all $\delta$-subharmonic functions $w$ on $\Omega$ satisfying

$$
\text { (1) }|w| \leqq M \quad \text { and (2) } \Phi_{w} \leqq \xi
$$

is compact.

One of the simplest choices for the function $\xi$ is

$$
\xi(r)=A r^{\kappa},
$$

where $A$ and $\kappa$ are positive constants. This is also one of the most important choices, since the case of $\kappa=2$ results in absolute continuity of the mass distributions. ${ }^{4}$

THEOREM 3. Let $M$ and $M^{\prime}$ be non-negative constants, and let $\mathcal{F}$ be $a$ family of $\delta$-subharmonic functions $w$ on $\Omega$ having mass distributions given by density functions $\rho_{w}$. If the functions $w$ satisfy

1 The same holds for $k>2$, but (as can be seen from the proof of Theorem 3) the mass distributions then vanish identically. 
(1) $|w| \leqq M$ and (2) $\left|\rho_{w}\right| \leqq M^{\prime}$ almost everywhere,

then $\mathcal{F}$ is normal. If, further, $\mathcal{F}$ contains all functions satisfying (1) and (2), then $\mathcal{F}$ is compact.

Proof. From (2) we have $\Phi_{v}^{2}(r) \leqq \pi M^{\prime} r^{2}$, and the normality is obvious. Moreover, by (3) of Theorem 2 this inequality persists when $w$ is taken as the limit of any sequence of functions in $\mathcal{F}$. It follows easily that the limit functions are absolutely continuous and that their density functions satisfy (2). Hence, the family of all functions satisfying (1) and (2) is compact.

An alternative approach to the compactness assertion involves the use of the Blaschke difference operator $\Delta_{r}^{B}[3$, p. 335] for a limit function $w$. From (2.1) and Theorem 2 we have

$$
\left|\Delta_{r}^{B} w(z)\right|=\frac{4}{r^{2}}\left|\mu_{r} w(z)-w(z)\right| \leqq \frac{4}{r^{2}} \Psi_{w}(r) \leqq 2 \pi M^{\prime} .
$$

The results of $\S 3$ of [5] show that this forces the mass distribution for $w$ to be absolutely continuous and that

$$
\left|\rho_{w}\right|=\frac{1}{2 \pi}\left|\Delta^{B} w\right| \leqq M^{\prime}
$$

holds almost everywhere.

By taking $\kappa=1$ in $(2.6)$ and applying the argument employed in the proof of Theorem 3, one arrives at an analogous theorem for linear mass distributions.

Theorem 4. Let $M$ and $M^{\prime}$ be non-negative constants, let $\Omega$ be a region intersecting the real axis $X$, and let $\mathcal{F}$ be a family of $\delta$-subharmonic functions on $\Omega$ having mass distributions on $X$ given by linear density functions $\lambda_{w}$. If the functions $w$ satisfy

(1) $|w| \leqq M$ and (2) $\left|\lambda_{w}\right| \leqq M^{\prime}$ almost everywhere on $X$,

then $\mathcal{F}$ is normal. If, further, $\mathcal{F}$ contains all functions satisfying (1) and (2), then $\mathcal{F}$ is compact.

Conditions for normality and compactness involving the $L^{p}$ norm will be given in $\$ 3$. Moreover, it is well to bear in mind, in connection with all of these conditions, that normality is a local property; if the conditions for normality are satisfied on any collection of regions covering $\Omega$, then the given family is normal on $\Omega$. Corresponding remarks apply to the various compactness conditions. For example, in Corollary 2.1 the constant $M$ and the function $\eta$ can be specified independently for each region in the covering collection. 
3. $L^{p}$ spaces of $\delta$-subharmonic functions. It is well known $[10$, p. 268] that for harmonic functions convergence in the norm of $L^{p}$ $(p \geqq 1)$ implies uniform convergence on compact subsets. The same situation occurs in the $\delta$-subharmonic case provided $\Psi \mathcal{F}(r)$ has limit zero.

THEOREM 5. Let $\mathcal{F}$ be a family of $\delta$-subharmonic functions on $\Omega$ such that $\lim _{r \rightarrow 0} \Psi \mathcal{F}(r)=0$. If $\left\{w_{n}\right\}$ is a sequence of functions in $\mathcal{F}$ converging in the $L^{p}$ sense $(p \geqq 1)$ on $\Omega$, then $\left\{w_{n}\right\}$ converges uniformly on compact subsets of $\Omega$.

In this proof, and for the rest of the paper, we shall use \|\|$_{p}$ to denote the $L^{p}$ norm over $\Omega$. Invoking (2.2), (2.3), and the Hölder inequality, we obtain

$$
\left|w_{j}(z)-w_{k}(z)\right| \leqq\left(\pi r^{2}\right)^{-1 / p}\left\|w_{j}-w_{k}\right\|_{p}+2 \Psi \mathfrak{F}(r)
$$

for $z$ on $\Omega_{r}$, and the theorem follows.

The similar inequality

$$
|w(z)| \leqq\left(\pi r^{2}\right)^{-1 / p}\|w\|_{p}+\Psi \mathcal{F}(r)
$$

leads to

THEOREM 6. Let $\mathcal{F}$ be a family of $\delta$-subharmonic functions on $\Omega$ such that $\lim _{r \rightarrow 0} \Psi \mathcal{F}(r)=0$. If $\mathcal{F}$ is a bounded subset of $L^{p}(p \geqq 1)$, then $\mathcal{F}$ is normal.

In fact, since $\mathcal{F}$ is bounded on compact subsets of $\Omega$, all the conclusions of Theorem 1 apply.

One way of effecting $\lim _{r \rightarrow 0} \Psi \mathcal{f}(r)=0$ is by assuming the mass distributions to be absolutely continuous and requiring the density functions to form a bounded subset of $L^{p}$ for some $p>1$.

THEOREM 7. Let $\mathcal{F}$ be a family of $\delta$-subharmonic functions $w$ on $\Omega$ having mass distributions given by density functions $\rho_{w}$ in $L^{p}(p>1)$. Then

(1) there is a positive constant $r(p)$, depending only on $p$, such that for $0<r<r(p)$

$$
\Psi_{w}(r) \leqq\left\|\rho_{w}\right\|_{p}(2 \pi r)^{1-1 / p}
$$

holds for all w in $\mathcal{F}$; and

(2) $\left\{\rho_{w}: w \in \mathcal{F}\right\}$ bounded in $L^{p}$ implies $\lim _{r \rightarrow 0} \Psi \mathcal{F}(r)=0$.

Proof. Hölder's inequality with $q=p /(p-1)$ yields

$$
\int_{N_{r}(z)}\left|\log \frac{r}{|z-\zeta|} \rho_{w}(\zeta)\right| d a(\zeta) \leqq\left\|\rho_{w}\right\|_{p}\left[2 \pi \int_{0}^{r} t\left(\log \frac{r}{t}\right)^{q} d t\right] 1 / q
$$


where $a$ denotes Lebesgue measure. We then choose $r(p)$ small enough so that

$$
t\left(\log \frac{r}{t}\right)^{q} \leqq t\left(\log \frac{1}{t}\right)^{q} \leqq 1
$$

for $0<t \leqq r<r(p)$. Hence,

$$
\Psi_{w}(r)=\sup _{z \in \Omega_{r}} \int_{N_{r}(z)} \log \frac{r}{|z-\zeta|}\left|\rho_{w}(\zeta)\right| d a(\zeta) \leqq\left\|\rho_{w}\right\|_{p}(2 \pi r)^{1 / q},
$$

proving (1) and thereby (2).

The essential ideas of the above proof are due to Myškis [13], who used them to establish a special case of the combination of Theorems 5 and 7. More recently, Frostman [9, pp. 92-93] has employed similar techniques to obtain an equicontinuity theorem for the $\alpha$-potentials of M. Riesz.

In the light of the preceding results it seems natural to introduce as norm the sum of the $L^{p}$ norms of $w$ and $\rho_{w}$. Accordingly, we frame

Definition 1 . Let $\mathcal{L}^{p}$, for $p>1$, be the set of all $\delta$-subharmonic functions $w$ on $\Omega$ with mass distributions given by density functions $\rho_{w}$ such that $\|w\|_{p}+\left\|\rho_{w}\right\|_{p}<+\infty$.

Clearly, $\mathcal{L}^{p}$ is a linear space under pointwise addition and multiplication by reals. It becomes a normed linear space when we set $\|w\|=\|w\|_{p}+\left\|\rho_{w}\right\|_{p}$. However,

$$
\|w\|=\left[\left(\|w\|_{p}\right)^{p}+\left(\left\|\rho_{w}\right\|_{p}\right)^{p}\right]^{1 / p},
$$

which defines the same topology, seems somewhat preferable in the important case of $p=2$, and we therefore agree to regard $\mathcal{L}^{p}$ as the normed linear space obtained using this norm. ${ }^{5}$

Collecting the information now available yields

Theorem 8. (1) If $\mathcal{Z}$ is a bounded subset of $\mathcal{L}^{p}$, the conclusions of Theorem 1 hold. (2) If $\left\{w_{n}\right\}$ is a sequence converging in $\mathcal{L}^{p}$ to a function $w$ in $\mathcal{L}^{p}$, then $\left\{w_{n}\right\}$ converges to $w$ uniformly on compact subsets of $\Omega$, and the conclusions of Theorem 2 hold.

Both of these assertions are evident consequences of Theorems 6 and 7 , coupled with the inequality

$$
|w(z)| \leqq M_{r}\|w\| \quad\left(w \in \mathcal{L}^{p}, z \in \Omega_{r}\right),
$$

where

$$
M_{r}=\left(\pi r^{2}\right)^{-1 / p}+(2 \pi r)^{1-1 / p} .
$$

5 The case of $p=2$ for $C^{\prime \prime}$ functions satisfying certain special boundary conditions on regions $\Omega$ admitting a Green's function has been studied by Aronszajn [2, p. 407]. 
Theorem 9. $\mathcal{L}^{p}$ is a separable, uniformly convex Banach space.

Proof. To show completeness, let $\left\{w_{n}\right\}$ be a Cauchy sequence in $\mathcal{L}^{p}$. Then by (3.4), $\left\{w_{n}\right\}$ converges uniformly on compact subsets of $\Omega$ to a function $w$, which is obviously also the $L^{p}$ limit of $\left\{w_{n}\right\}$. Moreover, since $\left\{w_{n}\right\}$ is bounded in $\mathcal{L}^{p}, w$ is a continuous $\delta$-subharmonic function.

Now, let $m_{n}$ and $m$ be the mass distributions for $w_{n}$ and $w$, respectively. In view of the vague convergence of $\left\{m_{n}\right\}$ to $m$, we have

$$
\int_{\sigma} f d m=\lim _{n \rightarrow \infty} \int_{\sigma} f d m_{n}
$$

where $\sigma$ is any closed disc lying in $\Omega$ and $f$ is any continuous function vanishing on the boundary of $\sigma$. Also, the completeness of $L^{p}$ ensures that $\left\{\rho_{w_{n}}\right\}$ converges in the $L^{p}$ sense on $\Omega$ to some function $\rho$. By the resulting $L^{1}$ convergence on $\sigma$ there follows

$$
\int_{\sigma} f \rho d a=\lim _{n \rightarrow \infty} \int_{\sigma} f \rho_{w_{n}} d a
$$

so that

$$
\int_{\sigma} f d m=\int_{\sigma} f \rho d a .
$$

The mass distribution for $w$ is thus given by the density function $\rho$. This implies that $w$ is in $\mathcal{L}^{p}$ and is the strong limit of $\left\{w_{n}\right\}$, establishing completeness.

Separability and uniform convexity are immediate from the corresponding properties of $L^{p}$.

With the aid of the theory of distributions it is easy to show that $\mathcal{L}^{p}$ is simply the completion of the space of all infinitely differentiable functions $\phi$ on $\Omega$ satisfying

$$
\int_{\Omega}\left(|\phi|^{p}+|\Delta \phi|^{p}\right) d a<+\infty .^{6}
$$

THEOREM 10. The subspace consisting of all infinitely differentiable functions of finite norm is dense in $\mathcal{L}^{p}$.

Proof. Let $w$ be an arbitrary function in $\mathcal{L}^{p}$, extended throughout the plane so as to vanish outside $\Omega$. If $\beta$ is any infinitely differentiable function having compact support, then the function $\beta * w$ formed by

- This leads at once to an explicit representation of the completion of the spaces considered by Aronszajn in [2, p. 407]. 
convolution is infinitely differentiable $[17$, p. 22] and satisfies $\Delta *(\beta * w)=\beta *(\Delta * w)$, where $\Delta$ denotes the generalized Laplacian of Schwartz (see [17, pp. 14-15]). Hence,

$$
\rho_{\beta * w}=\beta * \rho_{w} \text { almost everywhere on } \Omega .^{7}
$$

There remains but to take a sequence $\left\{\beta_{n}\right\}$ of non-negative infinitely differentiable functions having integrals equal to one and supports converging uniformly to the origin, since it is evident [17, pp. 22-23] that $\left\{\beta_{n} * w\right\}$ converges to $w$ in the metric of $\mathcal{L}^{p}$.

The theory of the spaces $\mathcal{L}^{p}$ should prove of interest in connection with the equation

$$
2 \pi \rho_{w}=\lambda w,
$$

where $\lambda$ is a given bounded measurable function on $\Omega$ and we seek as solutions $\delta$-subharmonic functions $w$ with mass distributions having density $\rho_{w}$. This, of course, generalizes the membrane equation

$$
\Delta w+\lambda w=0,
$$

since any $C^{\prime \prime}$ solution of the latter is a $\delta$-subharmonic function whose mass distribution is given by the density function $-\Delta w / 2 \pi$. An important aspect of the study of the generalized equation is that when $w$ satisfies (3.5), the $\mathcal{L}^{p}$ norm defines the same topology as the $L^{p}$ norm; in fact, if $M$ is an upper bound for $|\lambda|$ on $\Omega$, then

$$
\|w\|_{p} \leqq\|w\| \leqq\left[1+\left(\frac{M}{2 \pi}\right)^{p}\right]^{1 / p}\|w\|_{p} .
$$

Thus, the normal family properties of $\mathcal{L}^{p}$ spaces are available here when the usual $L^{p}$ norm $(p>1)$ is used.

As has been pointed out by Aronszajn $[1$, p. 138], continuity of the point functionals on a Hilbert space of functions implies ${ }^{8}$ the existence of a reproducing kernel-a fact which follows at once from the Riesz representation theorem for continuous linear functionals. It is thereby apparent from (3.4) that $\mathcal{L}^{2}$ admits a reproducing kernel.

The classical function-theoretic approach to the reproducing kernel, as developed by Bergman [6] and others, is based on extremal methods playing essentially the same role as the Riesz theorem. These extremal methods, which of course depend on continuity of

${ }^{7}$ An appropriate choice of $\beta$ yields results of Thompson [18] and Reade [15] on the mass distributions for areal means of potentials.

- Actually, Aronszajn's theorem is that continuity of the point functionals is equivalent to the existence of a reproducing kernel. 
the point functionals, have applications to certain interpolation problems and are of interest even when $p \neq 2$. For this reason we sketch briefly a derivation of this sort, in the setting of $\mathcal{L}^{2}$.

For any $z$ in $\Omega$ let $E_{z}=\left\{w \in \mathcal{L}^{2}: w(z)=1\right\}$. Obviously $E_{z}$ is convex, and (3.4) ensures that it is closed. Moreover, $E_{z}$ is non-empty, inasmuch as it is an easy matter to find a $C^{\prime \prime}$ function supported by an arbitrary neighborhood of $z$ and attaining the value 1 at $z$. There is therefore a unique element $w_{z}$ of $E_{z}$ having minimum norm.

The function $w_{z}$ so obtained has the properties (1) $w_{z}(z)=1$ and (2) $\left(w_{z}, \phi\right)=0$ for all functions $\phi$ in $\mathcal{L}^{2}$ vanishing at $z$. Assertion (2) is an immediate consequence of the minimality of $w_{z}$, since two vectors $p, q$ of a Hilbert space are orthogonal if and only if $\|p+\lambda q\| \geqq\|p\|$ holds for all scalars $\lambda$. Now, taking $w$ as an arbitrary function in $\mathcal{L}^{2}$ and setting $\phi=w(z) w_{z}-w$, we see that the function $K_{z}$ defined by

$$
K_{z}=\frac{w_{z}}{\left\|w_{z}\right\|^{2}}
$$

yields

$$
w(z)=\left(K_{z}, w\right) .
$$

Hence, $K_{z}$ is the reproducing kernel for $\mathcal{L}^{2}$.

4. Further remarks. It has been shown by Montel $[12$, p. 38] that the upper envelope of a normal family of continuous subharmonic functions is itself a continuous subharmonic function. This suggests the following question, which we leave unanswered. Is the upper envelope of the family $\mathcal{F}$ of Theorem 1 a $\delta$-subharmonic function? (The argument used by Montel proves that it is continuous.)

Let us consider now the family of elliptic equations

$$
\Delta w+\lambda w=0
$$

obtained by fixing constants $M>m>0$ and taking $\lambda$ as an arbitrary continuous function such that

$$
m \leqq \lambda \leqq M .
$$

If $\mathcal{F}$ is the family of all solutions of equations (4.1) satisfying

$$
\int_{\Omega}\left[(\operatorname{grad} w)^{2}+\lambda w^{2}\right] d a \leqq 1,
$$

then, as Bergman and Schiffer have proved [8, pp. 548-551] by means of the kernel function, $\mathcal{F}$ is normal. We remark that the normality of $\mathcal{F}$ is also a consequence of our results on $\mathcal{L}^{p}$, and that normality per- 
sists when (4.2) is replaced by the $L^{p}$ boundedness condition $\|w\|_{p} \leqq 1$, $p>1$.

\section{BIBLIOGRAPHY}

1. N. Aronszajn, La thérie des noyaux reproduisants et ses applications: première partie, Proc. Cambridge Philos. Soc. vol. 39 (1943) pp. 133-153.

2. - Green's functions and reproducing kernels, Proceedings of the Symposium on Spectral Theory and Differential Problems, 1951, pp. 355-411.

3. M. G. Arsove, Functions representable as differences of subharmonic functions, Trans. Amer. Math. Soc. vol. 75 (1953) pp. 327-365.

4. - Functions of potential type, Trans. Amer. Math. Soc. vol. 75 (1953) pp. $526-551$.

5. - The Looman-Menchoff theorem and some subharmonic function analogues, Proc. Amer. Math. Soc. vol. 6 (1955) pp. 94-105.

6. S. Bergman, The kernel function and conformal mapping, Mathematical Surveys, vol. 5, American Mathematical Society, 1950.

7. - Functions of the extended class in the theory of functions of several complex variables, Trans. Amer. Math. Soc. vol. 63 (1948) pp. 423-447.

8. S. Bergman and M. Schiffer, Kernel functions in the theory of partial differential equations of elliptic type, Duke Math. J. vol. 15 (1948) pp. 535-566.

9. O. Frostman, Distributions de masses normées par la métrique de $L^{p}$, Lund Univ. Math. Sem. supplementary vol. dedicated to Marcel Riesz (1952) pp. 90-100.

10. O. D. Kellog, Potential theory, Berlin, 1929.

11. P. Montel, Sur quelques familles de fonctions harmoniques, Fund. Math. vol. 25 (1935) pp. 388-407.

12. - Sur les fonctions convexes et les fonctions sousharmoniques, J. de Math. vol. 7 (1928) pp. 29-60.

13. A. D. Myskis, $A$ theorem on the convergence of a sequence of functions, Uspehi Matemati Ceskih Nauk vol. 7 (1952) pp. 186-190 (Russian).

14. T. Rad6, Subharmonic functions, Berlin, 1937.

15. M. O. Reade, On averages of Newtonian potentials, Bull. Amer. Math. Soc. vol. 53 (1947) pp. 321-331.

16. S. Saks and A. Zygmund, Analytic functions, Warsaw, 1952.

17. L. Schwartz, Theorie des distributions, vol. II, Actualités Scientifiques et Industrielles, no. 1122, 1951.

18. J. M. Thompson, Distributions of mass for averages of Newtonian potential functions, Bull. Amer. Math. Soc. vol. 41 (1935) pp. 744-752.

UNIVERSITY OF WASHINGTON 